\title{
Trombosis de válvulas protésicas biológicas
}

\author{
Dres. Amparo Fernández¹, Pablo Asadurian¹, Fernando Kuster², Víctor Dayan¹
}

\section{Resumen}

La trombosis de la válvula protésica biológica (TVPB) era considerada una entidad relativamente rara. Sin embargo, debido al aumento del uso de bioprótesis en cirugía cardíaca y al advenimiento de las prótesis biológicas transcatéter, ha adquirido mayor interés al ser reconocida como causa de disfunción protésica. Aún no se ha establecido la relevancia a largo plazo de la trombosis subclínica, ni están definidas las estrategias terapéuticas óptimas para prevenir la TVPB ni las complicaciones tromboembólicas. En esta revisión se analizan la fisiopatología, el diagnóstico y el tratamiento de la TVPB para contribuir al conocimiento de esta patología.

Palabras clave: TROMBOSIS

BIOPRÓTESIS

VÁLVULA AÓRTICA

REEMPLAZO DE LA VÁLVULA AÓRTICA TRANSCATÉTER

TROMBOEMBOLIA

ANTICOAGULANTES

\section{Biological prosthetic valve thrombosis}

\section{Summary}

Bioprosthetic valve thrombosis was considered a relatively rare entity. However, due to the increased use of bioprostheses in cardiac surgery and the advent of transcatheter biological prostheses, it has acquired more attention as a cause of prosthetic dysfunction. The long-term relevance of subclinical thrombosis has not yet been elucidated, nor are the optimal therapeutic strategies to prevent bioprosthetic valve thrombosis or thromboembolic complications. This review aims to provide a summary of the pathophysiology, diagnosis and treatment of bioprosthetic valve thrombosis to contribute to the knowledge of this pathology.

Key words: $\quad$ THROMBOSIS

BIOPROSTHESIS

AORTIC VALVE

TRANSCATHETER AORTIC VALVE REPLACEMENT

THROMBOEMBOLISM

ANTICOAGULANTS

\section{Trombose da válvula protética biológica}

\section{Resumo}

A trombose de prótese biológica foi considerada uma entidade relativamente rara. No entanto, devido ao aumento do uso de biopróteses em cirurgia cardíaca e o advento de próteses biológicas transcateter, tornou-se mais interessante como causa de disfunção protética. A relevância a longo prazo da trombose subclínica ainda não foi elucidada, nem são definidas as estratégias terapêuticas ideais para prevenir a trombose de prótese biológica ou complicações tromboembólicas. Esta revisão tem como objetivo fornecer um resumo da fisiopatologia, diagnóstico e tratamento da trombose de prótese biológica para contribuir o conhecimento desta patologia

Palavras chave: TROMBOSE

BIOPRÓTESE

VALVA AÓRTICA

SUBSTITUIÇÃO DA VALVA AÓRTICA TRANSCATETER

TROMBOEMBOLIA

ANTICOAGULANTES

1. Instituto Nacional de Cirugía Cardíaca. Montevideo, Uruguay.

2. Servicio Médico Integral. Montevideo, Uruguay.

Correspondencia: Dra. María Amparo Fernández. Gonzalo Ramírez 2094, apto 202. Montevideo, Uruguay.

Correo electrónico: ampiblu@gmail.com

Los autores declaran no tener conflictos de intereses.

Recibido Feb 11, 2020; aceptado Abr 20, 2020. 


\section{Introducción}

La sustitución de una válvula cardíaca nativa por una protésica conlleva un riesgo inherente de desarrollar ciertos procesos fisiopatológicos que pueden ocasionar disfunción protésica y la eventual necesidad de reintervención ${ }^{(1)}$. Existen cuatro etiologías principales de disfunción protésica: a) deterioro estructural (por ejemplo, por formación de pannus); b) deterioro no estructural; c) trombosis, y d) endocarditis. Estas entidades pueden intrincarse e incluso ocurrir simultáneamente. En efecto, el componente trombótico está presente de forma frecuente en la degeneración protésica o en la endocardi$\operatorname{tis}^{(2)}$.

A pesar de que las bioprótesis son menos trombogénicas que las prótesis mecánicas, la trombosis de la válvula protésica biológica (TVPB) es una entidad cada vez más reconocida como causa de disfunción valvular.

\section{Prevalencia e importancia del tema}

El interés en la TVPB ha surgido luego del reconocimiento de la trombosis subclínica diagnosticada por tomografía computada multicorte en válvulas protésicas biológicas implantadas tanto por vía transcatéter, como por cirugía convencional ${ }^{(3-5)}$. En un estudio de Hansson y colaboradores en válvulas aórticas implantadas transcatéter, 28 de 405 sujetos estudiados (7\%) cumplían con el diagnóstico tomográfico de trombosis ${ }^{(6)}$. Se trata de una complicación que puede ocurrir en las cuatro válvulas cardíacas, con incidencia y relevancia clínica en aumento por mayor empleo de bioprótesis en cirugía y rápida expansión del uso de válvulas bioprotésicas transcatéter ${ }^{(7)}$.

Si bien esta complicación se presenta en diversos tipos de bioprótesis, se observó una incidencia menor en aquellos pacientes con dosis terapéuticas de anticoagulación, comparados con los que no recibían anticoagulación, o eran tratados con dosis subóptimas, o solamente con antiagregantes plaqueta$\operatorname{rios}^{(3,6,8)}$.

La importancia radica en que se trata de una complicación potencialmente mortal que ha sido subdiagnosticada en los estudios más antiguos, posiblemente debido a la falta de criterios diagnósticos validados, con reportes de incidencia en torno al $0,1 \%-6 \%(9)$.

En un estudio del año 2015, Egbe y colaboradores ${ }^{(10)}$ constataron una incidencia de TVPB de 11,6 $\%$ en válvulas explantadas (considerando las cuatro válvulas cardíacas), con una incidencia de 10,9\%, para la válvula aórtica. Estas cifras apoyan la hipó- tesis de que probablemente la complicación haya sido subdiagnosticada en estudios previos.

Otro aspecto que hace desafiante el diagnóstico es la falta de una definición universal. En las guías para el reporte de mortalidad y morbilidad luego de intervenciones cardíacas ${ }^{(11)}$ del año 2008, la trombosis se definió como cualquier trombo no relacionado con infección, fijo a la valva protésica o en su proximidad, que ocluye el flujo sanguíneo, interfiere con la función valvular, o cuyo tamaño es tan grande que justifica su tratamiento. Esta definición deja de lado la trombosis subclínica, que también se cree, es más frecuente de lo anteriormente planteado.

\section{Fisiopatología}

Las prótesis biológicas quirúrgicas más usadas son xenoinjertos manufacturados a partir de tejido porcino o pericardio bovino, con o sin anillo (stented o stentless, respectivamente) ${ }^{(12)}$. Las válvulas transcatéter también consisten en tejido porcino o pericardio bovino montado sobre un stent metálico autoexpandible, o expandible con balón ${ }^{(13)}$. Todas las válvulas biológicas son menos trombogénicas que las válvulas mecánicas debido a que imitan mejor las propiedades hemodinámicas de las válvulas nativas, sin obstrucción al flujo sanguíneo central, y además poseen menor trombogenicidad intrínseca del material constitutivo(14). Las válvulas biológicas stentless son las menos trombogénicas ${ }^{(12)}$, aunque cualquier cuerpo extraño implantado en el organismo tiene potencial trombogénico.

Los mecanismos de trombosis protésica incluyen: a) malposición protésica; b) endotelización incompleta; c) presencia de flujo turbulento, y d) estasis sanguínea ${ }^{(2)}$. La influencia de los factores hemodinámicos se ve reflejada en el hecho de que la trombosis es más frecuente en las prótesis del lado derecho del corazón, donde la velocidad del flujo sanguíneo es menor ${ }^{(2)}$. Algunos de los factores favorecedores de trombosis serían particularmente relevantes en las válvulas transcatéter, como la aposición incompleta que puede crear áreas de estasis entre el anillo metálico y los tejidos nativos, promoviendo la formación local de trombos y retardando la endotelización ${ }^{(15)}$. También se ha visto que en este procedimiento los velos de la válvula nativa estenótica, que se desplazan lateralmente por el marco metálico de la prótesis, pueden desencadenar la formación de trombos ${ }^{(2)}$.

En un estudio, Mack y Holmes ${ }^{(16)}$ encontraron como factores predisponentes para TVPB: a) tamaño pequeño de la prótesis; b) índice de masa corporal (IMC) elevado; c) falta de anticoagulación, y d) 
implante de válvula transcatéter sobre prótesis quirúrgica previa. En una revisión de Sachdev y colaboradores ${ }^{(17)}$ se incluyeron como factores predictores, además de los ya nombrados, el sexo masculino, fibrilación auricular, dilatación de la aurícula, y estados de hipercoagulabilidad, entre otros. En el estudio de Hansson y colaboradores ${ }^{(6)}$ sobre 405 válvulas aórticas implantadas transcatéter, el tamaño mayor de la prótesis fue un factor predisponente para trombosis, mientras que el tratamiento con warfarina tuvo un efecto protector.

El riesgo de trombosis es mayor en los primeros tres meses posimplante debido a la ausencia de endotelización de la prótesis ${ }^{(12)}$. Como fue señalado más arriba, los distintos mecanismos de disfunción protésica pueden coexistir. Se plantea que la trombosis protésica, el pannus y la degeneración puedan ser parte de un mismo proceso patológico continuo, siendo la trombosis subclínica lo que ocurre más temprano, y la organización fibrótica lo que ocurre más tarde en la evolución ${ }^{(2)}$.

\section{Diagnóstico}

\section{Clínica}

La TVPB tiene una presentación clínica variable. La trombosis obstructiva se asocia con compromiso hemodinámico, síntomas de falla cardíaca y bajo gasto, a menudo con un progreso insidioso, que de diagnosticarse tardíamente puede conducir al shock cardiogénico. La trombosis no obstructiva puede ser asintomática (hallazgo imagenológico) o identificarse en la búsqueda etiológica de una embolia $^{(12)}$. La trombosis sintomática representa el extremo final del espectro de la TVPB, mientras que la trombosis subclínica de las valvas es mucho más frecuente ${ }^{(8)}$

A pesar de que clásicamente se consideraba que el riesgo de TVPB se limitaba a los primeros tres meses posteriores al implante, algunos estudios han demostrado que el pico de incidencia sería entre uno y dos años posimplante ${ }^{(18)}$.

El principal mecanismo de disfunción protésica es el deterioro estructural, pero la TVPB está siendo cada vez más reconocida como otra causa importante y potencialmente reversible ${ }^{(19)}$. En el escenario clínico puede ser complejo distinguir entre TVPB y degeneración protésica, aunque podría determinarse por la respuesta a la anticoagulación ${ }^{(8)}$.

\section{Ecocardiografía}

El ecocardiograma es el primer método de imagen para diagnosticar TVPB. El examen ecocardiográfico debe incluir múltiples vistas, con zoom para vi- sualizar los velos. Se debe realizar la medición de gradientes desde varias ventanas y calcular el área valvular para comparar con los estudios previos del mismo paciente y con tablas estándar para el tipo y tamaño de prótesis. Este proceso debe realizarse en condiciones normales de volemia y frecuencia cardíaca controlada ${ }^{(20)}$.

Debe considerarse la posibilidad de una TVPB en el ecocardiograma transtorácico (ETT) cuando los gradientes transvalvulares hayan aumentado más de $50 \%$ luego del implante, o en aquellos en que se observe un trombo recubriendo los velos protésicos en el lado "corriente abajo" (lado ventricular para las válvulas mitral y tricúspide; lado arterial para las válvulas aórtica y pulmonar)(21).

Siempre es importante considerar otros diagnósticos diferenciales que causan aumento de los gradientes transvalvulares, como la degeneración protésica, pannus, mismatch protésico o gasto cardíaco elevado. El trombo, en general, respeta los bordes de las valvas, a diferencia del pannus. Respecto a la calcificación, normalmente la presencia de calcificación mayor a leve se asocia con degeneración protésica más que con trombo, aunque su presencia no descarta un componente trombótico.

Otra alteración que puede encontrarse es la insuficiencia protésica por reducción en la movilidad de los velos y déficit de coaptación.

Todas estas alteraciones se resuelven habitualmente con tratamiento anticoagulante ${ }^{(21)}$.

En ocasiones pueden no visualizarse en forma óptima los velos por ETT y resultar compleja la diferenciación entre trombo, pannus o fibrosis. La sombra acústica debida a los metales de los soportes de las válvulas protésicas puede generar mayores dificultades $^{(20)}$. Cuando los hallazgos sean discordantes, o si la sospecha de TVPB persiste a pesar de un ETT negativo, debe realizarse un ecocardiograma transesofágico (ETE), especialmente para las prótesis en posición mitral o tricuspídea ${ }^{(21,22)}$.

El ETE permite evaluar la apariencia de las valvas, la motilidad y la función (obstrucción o regurgitación), por lo que es excelente para diagnosticar TVPB y debe recomendarse en la mayoría de los casos ante un ETT alterado ${ }^{(21)}$. La visualización de la prótesis aórtica puede ser desafiante, aun en el ETE. En caso de tener sombra del soporte de la bioprótesis aórtica deben realizarse vistas transgástricas con zoom ${ }^{(20)}$. Siempre que esté disponible, se sugiere utilizar ETE 3D ${ }^{(23)}$. En caso de que el ETE sea dudoso debe considerarse realizar una tomografía computada multidetector (TCMD).

Egbe y colaboradores ${ }^{(10)}$ proponen un algoritmo diagnóstico basado en tres predictores ecocardiográficos: 1) aumento $\geq 50 \%$ de los gradientes proté- 
sicos luego de cinco años de implantada la válvula (en ausencia de estado de alto gasto cardíaco); 2 ) aumento en el grosor de las valvas ( $>2 \mathrm{~mm}$ ), y 3) movimiento anormal de las valvas. En su estudio, realizaron un modelo de riesgo de estas tres características ecocardiográficas sumadas a fibrilación auricular paroxística e INR subterapéutico en pacientes anticoagulados con antagonistas de la vitamina $\mathrm{K}$ (AVK) y encontraron una sensibilidad de 72\%, especificidad de $90 \%$, valor predictivo positivo de $78 \%$ y valor predictivo negativo de $87 \%{ }^{(10)}$. El diagnóstico de TVPB fue realizado de forma correcta en el ecocardiograma inicial solo en una minoría de pacientes: $5 \%$ usando ETT y $13 \%$ usando ETE $^{(10)}$.

El algoritmo para el abordaje de la TVPB, propuesto por Pislaru y colaboradores ${ }^{(21)}$, implica la vigilancia de rutina con ETT luego del implante de la prótesis, y cuando se encuentren gradientes aumentados $>50 \%$, valvas engrosadas o motilidad restringida, realizar un ETE. Si se sospecha TVPB en el ETE, proponen realizar una prueba con warfarina para revertir las alteraciones.

\section{Tomografía computada multidetector}

La TCMD permite evaluar la apariencia y el movimiento de las válvulas protésicas, además de detectar la presencia de trombo y sus características. Se ha demostrado que la TCMD agrega un valor adicional al ecocardiograma para el seguimiento de las bioprótesis $^{(24)}$.

Existen diferentes hallazgos tomográficos de la TVPB subclínica. En el estudio de Makkar y colaboradores ${ }^{(3)}$, esta se definió como una reducción mayor de 50\% del movimiento de al menos una de las valvas. En otro estudio, realizado por Pache y colaboradores $^{(25)}$, se describió un engrosamiento con hipoatenuación de las valvas, que fue atribuido a trombosis, dado que era completamente reversible con anticoagulación. Otros dos estudios definieron la TVPB subclínica en la TCMD como un engrosamiento hipoatenuado, con o sin rigidez de una o más valvas identificables en al menos dos proyecciones diferentes, y dos intervalos diferentes de tiempo de reconstrucción.

La TCMD es potencialmente mejor que el ecocardiograma para evaluar las válvulas aórtica y pulmonar, mientras que el ecocardiograma valoraría mejor las válvulas mitral y tricúspide.

\section{Tomografía por emisión de positrones}

Recientemente se describió que la tomografía por emisión de positrones con ${ }^{18} \mathrm{~F}$-fluoruro puede identificar precozmente aquellas bioprótesis con degeneración subclínica (antes que la tomografía computada y el ecocardiograma) ${ }^{(26)}$. Esto se debe a que el ${ }^{18} \mathrm{~F}$-fluoruro se une preferencialmente a zonas con microcalcificaciones indicativas de degeneración protésica ${ }^{(26)}$. Esta herramienta podría ser útil en predecir la disfunción protésica, seleccionando a aquellos pacientes con mayor riesgo de degeneración, en quienes se justificaría realizar un seguimiento más estrecho, o intervenciones terapéuticas tempranas. Adicionalmente, la señal con fluoruro se encontró no solo en las áreas de microcalcificaciones, sino también en bioprótesis con trombosis que luego demostraron mayor grado de disfunción protésica, lo que plantea que la trombosis valvular puede ser una diana terapéutica para prevenir la degeneración y disfunción protésicas ${ }^{(27)}$.

\section{Tratamiento}

Existe evidencia de que el tratamiento más efectivo para la TVPB, tanto clínica como subclínica, en pacientes hemodinámicamente estables, es la anticoagulación oral que ha demostrado regresión de los hallazgos, sin efectos adversos ${ }^{(8)}$, con una efectividad de los AVK de $83 \%$ para los pacientes con sospecha de TVPB, y una respuesta en tres meses de la mayoría de los pacientes ${ }^{(3,18,19)}$. La guía ACC/AHA 2017 recomienda iniciar tratamiento con AVK en pacientes en que se sospeche o confirme la trombosis de la bioprótesis y que estén hemodinámicamente estables, sin contraindicaciones para anticoagulación (recomendación IIa nivel de evidencia C) ${ }^{(28)}$.

También existe evidencia que respalda el uso de anticoagulación en las bioprótesis implantadas transcáteter. En un estudio de Del Trigo y colaboradores ${ }^{(29)}$ se incluyeron 2.466 pacientes con implante de válvula aórtica transcutánea, de los cuales $28,7 \%$ recibió anticoagulación oral (en su mayoría con AVK y algunos con anticoagulantes directos). Se encontró que la falta de anticoagulación se asoció significativamente con incremento de los gradientes transvalvulares y mayor riesgo de deterioro valvular hemodinámico, definido como un aumento $\geq 10$ $\mathrm{mmHg}$ del gradiente medio en el ecocardiograma al año.

A pesar de que se ha visto que la TVPB es menos frecuente en pacientes anticoagulados ${ }^{(3,8)}$, aún no se ha podido demostrar un efecto clínico beneficioso con anticoagulación de rutina en la sustitución valvular aórtica transcatéter (TAVR) o quirúrgica $\left(\right.$ SAVR) ${ }^{(30-32)}$. Debido a ello, en la guía AHA/ACC 2017, la indicación de anticoagulación con AVK para las bioprótesis durante un período de tres a seis meses (en pacientes con bajo riesgo de hemorragia) es una indicación clase IIa, nivel de evidencia $\mathrm{B}^{(28)}$. En la guía ESC 2017 el tratamiento anticoagulante con AVK puede considerarse luego de la SAVR por 
bioprótesis con indicación clase IIb, nivel de evidencia $\mathrm{C}^{(33)}$.

La única anticoagulación que ha demostrado ser beneficiosa es con AVK y no está claro aún el rol de los anticoagulantes directos. El ensayo controlado GALILEO $^{(34)}$, que comparaba rivaroxabán con doble antiagregación plaquetaria luego de TAVR, tuvo que ser detenido precozmente debido al aumento de la mortalidad por todas las causas y de los eventos hemorrágicos en los pacientes tratados con rivaroxabán comparado con doble antiagregación plaquetaria. Existen otros ensayos clínicos en desarrollo, como el estudio ATLANTIS(35), cuya hipótesis es la superioridad de apixabán sobre el tratamiento estándar recomendado para reducir el riesgo de las complicaciones tromboembólicas pos-TAVR, y un estudio danés en curso en el que se randomizan pacientes sometidos a sustitución valvular aórtica con bioprótesis a anticoagulación por seis meses con rivaroxabán versus antiagregación con ácido acetilsalicílico. (ClinicalTrials.gov Identifier: NCT02974920). Tendremos que esperar estos resultados y los de otros estudios para clarificar el rol de los anticoagulantes directos.

\section{Conclusiones}

La TVPB es una de las causas de degeneración valvular, clásicamente considerada como un fenómeno raro, pero que podría ser más frecuente de lo considerado; la ausencia de una definición única puede haber contribuido a este subdiagnóstico. La presencia de gradientes transprotésicos aumentados debe alertar al clínico sobre la posibilidad de estar frente a esta patología.

El advenimiento de la TAVR y el aumento del uso de las bioprótesis en SAVR, en conjunto con los hallazgos de trombosis subclínica tanto en TAVR como en SAVR, han despertado el interés en definir las estrategias terapéuticas óptimas para prevenir la TVPB y sus complicaciones tromboembólicas. Sin embargo, en la literatura actual no se encuentran recomendaciones basadas en la evidencia respecto al uso de anticoagulantes para prevenir la TVPB luego de la colocación de bioprótesis, especialmente en posición aórtica. El gran crecimiento en la realización y publicación de estudios respecto a la indicación de TAVR, no se acompañó paralelamente con la búsqueda de la terapia antitrombótica óptima que asegure buenos resultados a largo plazo, especialmente en lo que respecta a la TVPB. Hasta disponer de más estudios prospectivos, las guías sugieren utilizar AVK durante un tiempo corto (aproximadamente tres meses) luego de la SAVR y doble terapia antiplaquetaria luego de la TAVR.
Amparo Fernández, https://orcid.org/0000-0003-4243-7844 Pablo Asadurian, https://orcid.org/0000-0002-0959-0890 Fernando Kuster, https://orcid.org/0000-0003-1192-8675 Víctor Dayan, https://orcid.org/0000-0002-5470-0585

Este artículo fue aceptado para su publicación por: Editor jefe Dr. Gerardo Soca.

\section{Bibliografía}

1. Capodanno D, Petronio AS, Prendergast B, Eltchaninoff H, Vahanian A, Modine T, et al. Standardized definitions of structural deterioration and valve failure in assessing long-term durability of transcatheter and surgical aortic bioprosthetic valves: a consensus statement from the European Association of Percutaneous Cardiovascular Interventions (EAPCI) endorsed by the European Society of Cardiology (ESC) and the European Association for Cardio-Thoracic Surgery (EACTS). Eur J Cardiothorac Surg. 2017;52(3):408-17. doi:10.1093/ejcts/ezx244

2. Dangas GD, Weitz JI, Giustino G, Makkar R, Mehran R. Prosthetic heart valve thrombosis. J Am Coll Cardiol. 2016;68(24):2670-89. doi:10.1016/j.jacc. 2016.09.958

3. Makkar RR, Fontana G, Jilaihawi H, Chakravarty T, Kofoed KF, De Backer O, et al. Possible subclinical leaflet thrombosis in bioprosthetic aortic valves. N Eng J Med. 2015;373(21):2015-24. doi:10.1056/NEJMoa1509233

4. Pache G, Schoechlin S, Blanke P, Dorfs S, Jan$\operatorname{der} \mathbf{N}$, Arepalli CD, et al. Early hypo-attenuated leaflet thickening in balloon-expandable transcatheter aortic heart valves. Eur Heart J. 2016;37(28): 2263-71. doi:10.1093/eurheartj/ehv526

5. Laschinger JC, Wu C, Ibrahim NG, Shuren JE. Reduced leaflet motion in bioprosthetic aortic valves-The FDA perspective. N Eng J Med. 2015;373(21): 1996-8. doi:10.1056/NEJMp1512264

6. Hansson NC, Grove EL, Andersen HR, Leipsic J, Mathiassen ON, Jensen JM, et al. Transcatheter aortic valve thrombosis: incidence, predisposing factors, and clinical implications. J Am Coll Cardiol. 2016;68(19):2059-69. doi:10.1016/j.jacc.2016.08.010

7. Puri R, Auffret V, Rodes-Cabau J. Bioprosthetic valve thrombosis. J Am Coll Cardiol. 2017;69(17): 2193-211. doi:10.1016/j.jacc.2017.02.051

8. Chakravarty T, Søndergaard L, Friedman J, De Backer O, Berman D, Kofoed KF, et al. Subclinical leaflet thrombosis in surgical and transcatheter bioprosthetic aortic valves: an observational study. Lancet 2017;389(10087):2383-92. doi:10.1016/ s0140-6736 (17) $30757-2$ 
9. Grunkemeier GL, Rahimtoola SH. Artificial heart valves. Annu Rev Med. 1990;41:251-63. doi:10. 1146/annurev.me.41.020190.001343

10. Egbe AC, Pislaru SV, Pellikka PA, Poterucha JT, Schaff HV, Maleszewski JJ, et al. Bioprosthetic valve thrombosis versus structural failure: clinical and echocardiographic predictors. J Am Coll Cardiol. 2015;66(21):2285-94. doi:10.1016/j.jacc.2015.09.022

11. Akins CW, Miller DC, Turina MI, Kouchoukos NT, Blackstone EH, Grunkemeier GL, et al. Guidelines for reporting mortality and morbidity after cardiac valve interventions. J Thorac Cardiovasc Surg. 2008;135(4):732-8. doi:10.1016/ j.jtcvs.2007.12.002

12. Lim WY, Lloyd G, Bhattacharyya S. Mechanical and surgical bioprosthetic valve thrombosis. Heart 2017;103(24):1934-41. doi:10.1136/heartjnl-2017-311856

13. Taramasso M, Pozzoli A, Latib A, La Canna G, Colombo A, Maisano F, et al. New devices for TAVI: technologies and initial clinical experiences. Nat Rev Cardiol. 2014;11(3):157-67. doi:10.1038 nrcardio.2013.221

14. Sun JC, Davidson MJ, Lamy A, Eikelboom JW. Antithrombotic management of patients with prosthetic heart valves: current evidence and future trends. Lancet (London, England). 2009;374(9689):565-76. doi:10.1016/s0140-6736(09)60780-7

15. Mylotte D, Andalib A, Theriault-Lauzier P, Dorfmeister M, Girgis M, Alharbi W, et al. Transcatheter heart valve failure: a systematic review. Eur Heart J. 2015;36(21):1306-27. doi:10.1093/eurheartj/ehu388

16. Mack M, Holmes D. Bioprosthetic valve thrombosis: the harder one looks, the more one finds. J Thorac Cardiovasc Surg. 2016;152(4):952-3. doi:10.1016/ j.jtcvs.2016.07.024

17. Sachdev S, Bardia N, Nguyen L, Omar B. Bioprosthetic valve thrombosis. Cardiol Res. 2018;9(6): 335-42. doi:10.14740/cr789

18. Pislaru SV, Hussain I, Pellikka PA, Maleszewski JJ, Hanna RD, Schaff HV, et al. Misconceptions, diagnostic challenges and treatment opportunities in bioprosthetic valve thrombosis: lessons from a case series. Eur J Cardiothorac Surg. 2015;47(4) 725-32. doi:10.1093/ejcts/ezu201

19. Egbe AC, Connolly HM, Pellikka PA, Schaff HV, Hanna R, Maleszewski JJ, et al. Outcomes of Warfarin therapy for bioprosthetic valve thrombosis of surgically implanted valves: a prospective study. JACC Cardiovasc Interv. 2017;10(4):379-87. doi:10.1016/j.jcin.2016.11.027

20. Stewart WJ. Thrombosis of bioprosthetic valves: can we afford to ignore it? J Am Coll Cardiol 2015;66(21):2295-7. doi:10.1016/j.jacc.2015.09.082

21. Pislaru SV, Pellikka PA, Schaff HV, Connolly HM. Bioprosthetic valve thrombosis: the eyes will not see what the mind does not know. J Thorac Cardiovasc Surg. 2015;149(6):e86-7. doi:10.1016/j.jtcvs. 2015.03.012

22. Daniel WG, Mugge A, Grote J, Hausmann D, Nikutta P, Laas J, et al. Comparison of transthoracic and transesophageal echocardiography for detection of abnormalities of prosthetic and bioprosthetic valves in the mitral and aortic positions. Am J Cardiol. 1993;71(2):210-5.

23. Özkan M, Gürsoy OM, Astarcioglu MA, Gündüz S, Çakal B, Karakoyun S, et al. Real-time three-dimensional transesophageal echocardiography in the assessment of mechanical prosthetic mitral valve ring thrombosis. Am J Cardiol. 2013;112(7):977-83. doi:10.1016/j.amjcard.2013.05.032

24. Leetmaa T, Hansson NC, Leipsic J, Jensen K, Poulsen SH, Andersen HR, et al. Early aortic transcatheter heart valve thrombosis: diagnostic value of contrast-enhanced multidetector computed tomography. Circ Cardiovasc Interv. 2015;8(4). pii: 001596. doi:10.1161/circinterventions.114.001596

25. Pache G, Schoechlin S, Blanke P, Dorfs S, Jander N, Arepalli CD, et al. Early hypo-attenuated leaflet thickening in balloon-expandable transcatheter aortic heart valves. Eur Heart J. 2016;37(28): 2263-71. doi:10.1093/eurheartj/ehv526

26. Cartlidge TRG, Doris MK, Sellers SL, Pawade TA, White AC, Pessotto R, et al. Detection and prediction of bioprosthetic aortic valve degeneration. J Am Coll Cardiol. 2019;73(10):1107-19. doi:10.1016/ j.jacc.2018.12.056

27. Fayad ZA, Calcagno C. Sodium fluoride PET and aortic bioprosthetic valve degeneration: implications for patient diagnosis, management, and treatment. J Am Coll Cardiol. 2019;73(10):1120-2. doi:10.1016/ j.jacc.2018.12.057

28. Nishimura RA, Otto CM, Bonow RO, Carabello BA, Erwin JP 3rd, Fleisher LA, et al. 2017 AHA/ACC Focused update of the 2014 AHA/ACC guideline for the management of patients with valvular heart disease: a report of the American College of Cardiology/American Heart Association task force on clinical practice guidelines. J Am Coll Cardiol. 2017;70(2):252-89. doi:10.1016/j.jacc.2017.03.011

29. Del Trigo M, Muñoz-García AJ, Latib A, Auffret V, Wijeysundera HC, Nombela-Franco L, et al. Impact of anticoagulation therapy on valve haemodynamic deterioration following transcatheter aortic valve replacement. Heart 2018;104(10):814-20. doi:10 . 1136/heartjnl-2017-312514

30. Chakravarty T, Patel A, Kapadia S, Raschpichler M, Smalling RW, Szeto WY, et al. Anticoagulation after surgical or transcatheter bioprosthetic aortic valve replacement. J Am Coll Cardiol. 2019;74(9): 1190-200. doi:10.1016/j.jacc.2019.06.058 
31. Aramendi JI, Mestres CA, Martinez-Leon J, Campos V, Munoz G, Navas C. Triflusal versus oral anticoagulation for primary prevention of thromboembolism after bioprosthetic valve replacement (trac): prospective, randomized, co-operative trial. Eur J Cardiothorac Surg. 2005;27(5):854-60. doi:10.1016/j.ejcts.2004.12.064

32. Rafiq S, Steinbrüchel DA, Lilleør NB, Møller CH, Lund JT, Thiis JJ, et al. Antithrombotic therapy after bioprosthetic aortic valve implantation: Warfarin versus aspirin, a randomized controlled trial. Thromb Res. 2017;150:104-10. doi:10.1016/j. thromres.2016.11.021

33. Falk V, Baumgartner H, Bax JJ, De Bonis M, Hamm C, Holm PJ, et al. 2017 ESC/EACTS guide- lines for the management of valvular heart disease.

Eur J Cardiothorac Surg. 2017;52(4):616-64. doi:10.1093/ejcts/ezx324

34. Windecker S, Tijssen J, Giustino G, Guimarães AHC, Mehran R, Valgimigli M, et al. Trial design: Rivaroxaban for the prevention of major cardiovascular events after transcatheter aortic valve replacement: Rationale and design of the GALILEO study. Am Heart J. 2017;184:81-7. doi:10.1016/j.ahj. 2016.10.017

35. Collet JP, Berti S, Cequier A, Van Belle E, Lefevre T, Leprince $P$, et al. Oral anti-Xa anticoagulation after trans-aortic valve implantation for aortic stenosis: the randomized ATLANTIS trial. Am Heart J. 2018;200:44-50. doi:10.1016/j.ahj.2018.03.008 